\title{
Arterial Collapse during Thrombectomy for Stroke: Clinical Evidence and Experimental Findings in Human Brains and In Vivo Models
}

\author{
(D)Y. Liu, (D)D. Gebrezgiabhier, (D). Zheng, (D)A.J. Shih, (DN. Chaudhary, (D) A.S. Pandey, (D).L.A. Larco, (D) S.I. Madhani, \\ (D) M. Abbasi, (D) A.H. Shahid, (D) R.A. Quinton, (D) Kadirvel, (D) W. Brinjikji, (DD.F. Kallmes, and DL.E. Savastano
}

O- Dis

\begin{abstract}
BACKGROUND AND PURPOSE: Aspiration thrombectomy has become a preferred approach to recanalize large-vessel occlusion in stroke with a growing trend toward using larger-bore catheters and stronger vacuum pumps. However, the mechanical response of the delicate cerebral arteries to aspiration force has not been evaluated. Here, we provide preclinical and clinical evidence of intracranial arterial collapse in aspiration thrombectomy.
\end{abstract}

MATERIALS AND METHODS: We presented a clinical case of arterial collapse with previously implanted flow diverters. We then evaluated the effect of vacuum with conventional aspiration catheters (with and without stent retrievers) in a rabbit model ( $n=3$ ) using fluoroscopy and intravascular optical coherence tomography. Then, in a validated human cadaveric brain model, we conducted 168 tests of direct aspiration thrombectomy following an experimental design modifying the catheter inner diameter $(0.064$ inch, 0.068 inch, and 0.070 inch), cerebral perfusion pressures (mean around 60 and $90 \mathrm{~mm} \mathrm{Hg}$ ), and anterior-versus-posterior circulation. Arterial wall response was recorded and graded via direct transluminal observation.

RESULTS: Arterial collapse was observed in both the patient and preclinical experimental models. In the human brain model, arterial collapse was observed in $98 \%$ of cases in the $\mathrm{M} 2$ and in all the cases with complete proximal flow arrest. A larger bore size of the aspiration catheter, a lower cerebral perfusion pressure, and the posterior circulation in comparison with the anterior circulation were associated with a higher probability of arterial collapse.

CONCLUSIONS: Arterial collapse does occur during aspiration thrombectomy and is more likely to happen with larger catheters, lower perfusion pressure, and smaller arteries.

ABBREVIATIONS: $\mathrm{ACS}=$ arterial collapse score; $\mathrm{BA}=$ basilar artery; MAP = mean arterial pressure; $\mathrm{OCT}$ = optical coherence tomography

sing aspiration thrombectomy as the first-line approach to recanalize large-vessel occlusion in stroke has attracted increasing attention because direct aspiration is associated with a shorter recanalization time and lower cost, ${ }^{1}$ and several

Received May 12, 2021; accepted after revision October 4.

From the Departments of Radiology (Y.L., M.A., R.K., W.B., D.F.K.) and Neurosurgery (J.L.A.L., S.I.M., A.H.S., L.E.S.) and Division of Anatomic Pathology (R.A.Q.), Mayo Clinic, Rochester, Minnesota; Departments of Mechanical Engineering (Y.L., Y.Z., A.J.S.), Neurosurgery (D.G., A.S.P., L.E.S.), and Radiology (N.C.), University of Michigan, Ann Arbor, Michigan; UCSF Graduate Program in Bioengineering (D.G.), University of California Berkeley, San Francisco, California; and Department of Mechanical Engineering (Y.Z.), Worcester Polytechnic Institute, Worcester, Massachusetts. This work was supported by the National Institutes of Health National Institute of Neurological Disorders and Stroke grant No. NS105853.

Please address correspondence to Luis E. Savastano, MD, PhD, Department of Neurosurgery, Mayo Clinic, 200 First St SW, Rochester, MN 55905; e-mail: Savastano.Luis@mayo.edu; @SavastanoLuisMD

- Indicates open access to non-subscribers at www.ajnr.org

Indicates article with supplemental online videos.

http://dx.doi.org/10.3174/ajnr.A7389 randomized trials have shown noninferiority of direct aspiration compared with stent retrievers. ${ }^{2,3}$ Because higher rates of successful recanalization were seen with larger-catheter bores, ${ }^{4}$ current trends in catheter development support the use of the largest suction catheter that the vessel can accommodate to maximize clot extraction and minimize catheter clogging. In response to this paradigm, newer catheters under development and in the pipeline for commercialization have a larger inner diameter and stronger vacuum power. Although larger-bore catheters were reported to better ingest clots and achieve higher first-pass recanalization, ${ }^{1}$ their potential complications related to the increased aspiration flow remain unclear and future research is warranted. In this study, first, we reported a clinical case of arterial collapse detected in an artery previously treated with multiple telescoping flow diverters. Second, we provided further in vivo evidence of arterial collapse in a rabbit model using fluoroscopic visualization of stent retriever collapse and optical coherence tomography (OCT). Third, we conducted a comprehensive 

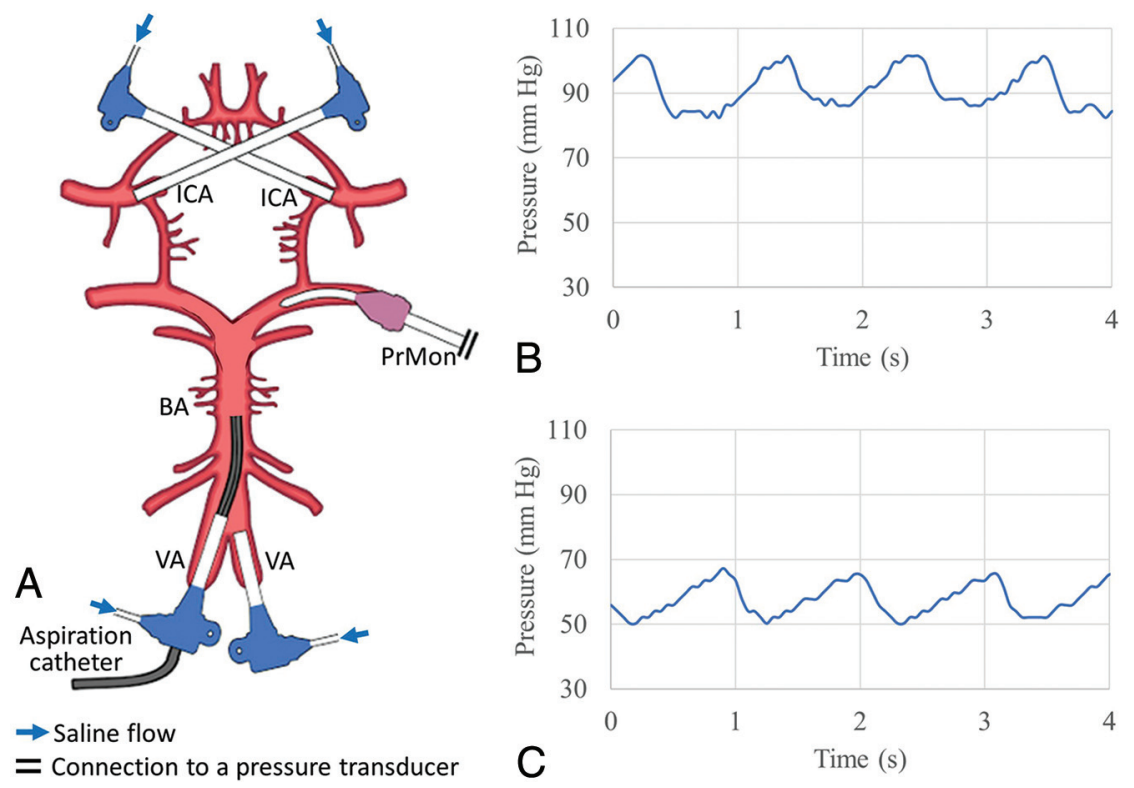

FIG 1. Whole human brain model setup. A, Both the ICA and the vertebral arteries (VA) were cannulated with $8 \mathrm{~F}$ sheaths, which were connected to a pump system to deliver saline flow at physiologically accurate rates. An aspiration catheter was inserted in the BA. Continuous intra-arterial pressure monitoring (PrMon) informed on high MAP (B) and low MAP $(C)$ conditions.

\section{Whole Human Brain Model}

The whole human brain model used in this study has been previously described. ${ }^{6,7}$ After institutional approval, fresh adult brains were harvested during research-consented postmortem examinations within 24 hours of death. Extra effort was made to preserve the circle of Willis and minimize damage to perforating arteries. Both ICAs and vertebral arteries were cannulated by $8 \mathrm{~F}$ sheaths and connected to a pump system to infuse $0.9 \%$ saline solution (Fig $1 A$ ). The pump system was adjusted to deliver physiologic flow rates of $\sim 310$ $\mathrm{mL} / \mathrm{min}$ for each ICA and $\sim 160 \mathrm{~mL} /$ min for each basilar artery (BA). Before each aspiration test, mean arterial pressure (MAP), defined as the mean of the systolic and diastolic pressure in the target arteries, was adjusted by modifying the resistance of the system by selectively clamping the small branching arteries with aneurysm clips

experimental study in a validated human brain model to analyze the mechanical response of cerebral arteries to different catheter sizes, perfusion pressures, and arteries.

\section{MATERIALS AND METHODS \\ Rabbit Model}

The rabbit model used in this study was previously described and was approved by the Institutional Animal Care and Use Committee (Mayo Clinic). ${ }^{5}$ Briefly, arterial access at the right carotid artery was obtained in adult New Zealand white rabbits $(n=3)$, and a $6 \mathrm{~F}$ Glidesheath Slender Sheath (Terumo) was advanced under fluoroscopy into the upper third of the thoracic aorta. Then, a 5F Sofia aspiration catheter (MicroVention) was navigated over an 014 Synchro microwire (Stryker) and a Marksman microcatheter (Medtronic) to the abdominal aorta. Then, a $6 \times 40 \mathrm{~mm}$ Solitaire stent retriever (Medtronic) was deployed with 4 radiopaque markers disposed in the arterial lumen, and the microcatheter was completely removed. The $5 \mathrm{~F}$ catheter was then connected to a 405 Gomco suction pump (Allied Healthcare) with $-650 \mathrm{~mm}$ $\mathrm{Hg}$ vacuum pressure, and the fluoroscopic changes in the radiopaque marker configuration were recorded. Five cycles of vacuum on/off were repeated in each animal. Then, the stent was removed from the animal, and a 2.7F OCT imaging catheter (Dragonfly OPTIS and OPTIS Integrated System; St. Jude Medical) was introduced into the aorta through the sheath and advanced distal to the 5F aspiration catheter. Under OCT observation, the aspiration catheter was connected to the same vacuum pump, and the changes in the vessel wall were recorded. Five cycles of vacuum on/off were repeated in each animal. as needed. To ensure patent leptomeningeal collateralization, we created a focal occlusion of the proximal MCA by strangulating the vessel with a vascular suture and measured the pressure distally and proximally. Pressure was measured by a transducer (15PDAA5 ASDX series; Honeywell). Aspiration catheters were then advanced into the target arteries and connected to an aspiration pump (405 Gomco). The arterial responses to the vacuum were recorded, and videos were analyzed by a team of engineers and neurointerventionalists.

We analyzed the effect of the following variables in relation to arterial wall response: 1) type of cerebral artery exposed to the vacuum: M1 and M2 segments of the MCA and BA; 2) bore size of suction catheters: 0.064 inch (ACE64; Penumbra), 0.068 inch (ACE68; Penumbra), and 0.070 inch (6F Sofia PLUS; MicroVention); 3) MAP within a physiologically relevant spectrum with a "high" mean MAP of 90 [SD, 5] mm Hg (Fig 1B), a "low" mean MAP of 60 [SD, 5] mm Hg (Fig $1 C$ ), and flow arrest in the target artery by interrupting flow in the corresponding sheath to mimic the clinical practice of inflating a balloon proximal to the occlusion to reduce distal embolization.

Six M1s, 6 M2s, and 7 BAs from 7 brains were tested, and a total of 171 aspiration tests were performed. For each brain, the ipsilateral M1 and M2 were tested to eliminate any effects of potential damage to the tested side.

\section{Statistical Analysis}

To predict the possibility of arterial collapse for different aspiration catheters, flow conditions, and aspiration locations, we performed binomial logistic regression. To decide which variable should be included in the regression model, $\chi^{2}$ tests were conducted, and variables with $P<.2$ were included in the regression model. 

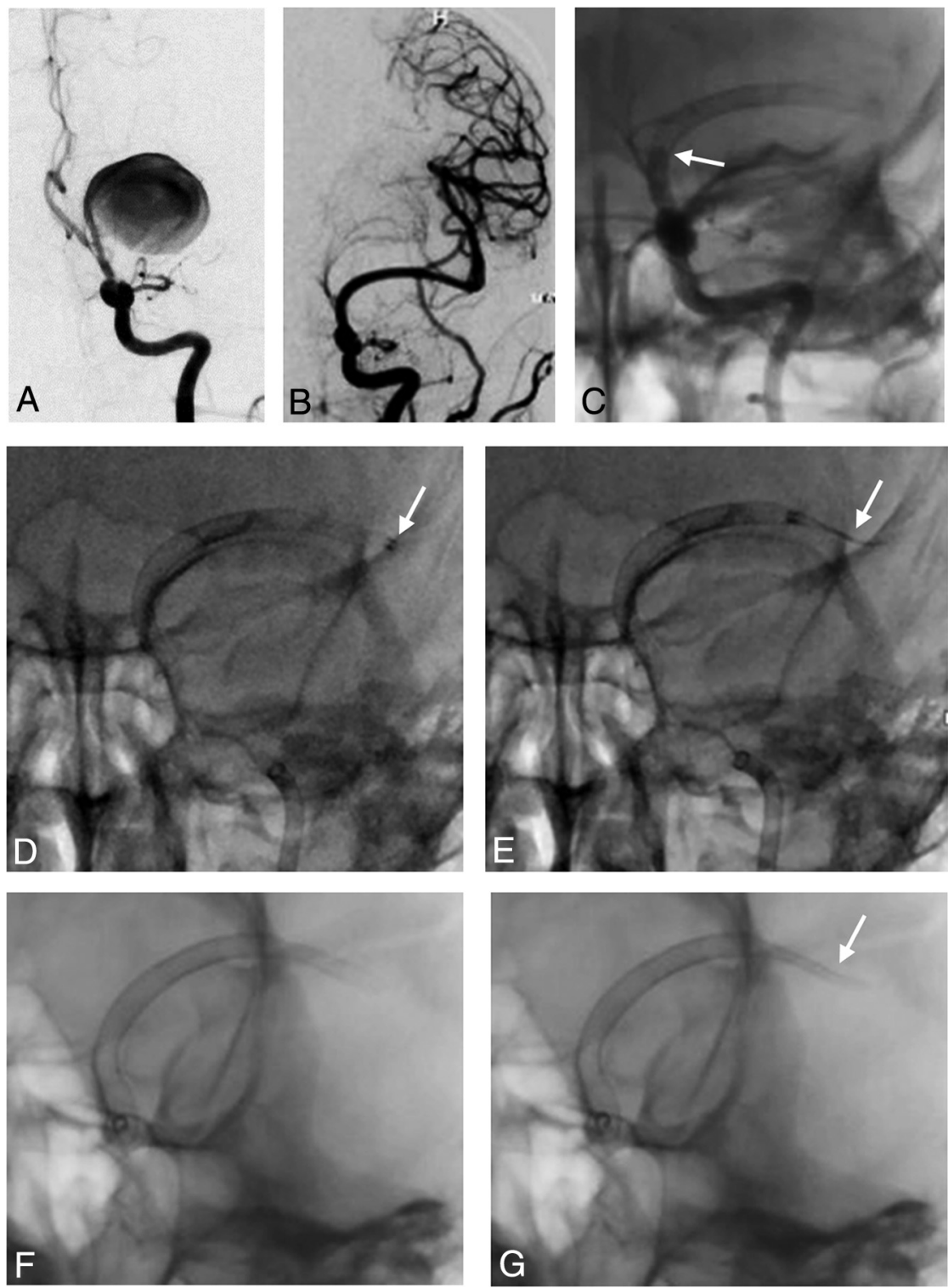

FIG 2. Clinical evidence of arterial collapse. A, Left ICA angiography in an anterior-posterior projection demonstrates a giant fusiform aneurysm in the left MCA. $B$, Left ICA angiogram after deployment of multiple telescoping flow diverters with acute occlusion of the aneurysm. $C$, Unsubtracted angiography of the left ICA obtained 5 days postprocedure shows in-stent thrombosis (arrow). D, Direct aspiration with a 068 catheter navigated distal to flow diverters (arrow). $E$, Severe collapse of the distal flow diverters on vacuum and device pull back (arrow). $F$ and $G$, Partial collapse of the distal telescoping flow diverters with a suction catheter connected to vacuum at the proximal cavernous ICA.

Statistical significance for the independent variables of the regression model was indicated by $P<.05$. The overall calibration error of the regression model was tested using the Hosmer-Lemeshow goodness of fit test. All statistical analyses were performed using SPSS, Version 25 (IBM).

\section{RESULTS}

\section{Clinical Case}

A 25-year-old woman with seizures and headaches was found to have a giant fusiform aneurysm in the left MCA (Fig 2A). Five flow diverters (Pipeline Embolization Device; Medtronic) were coaxially implanted from the cavernous ICA to the distal M1 segment of the MCA. Four devices were telescoped to reconstruct the parent artery at the level of the aneurysm, and an extra device was deployed distally to ensure good coverage across the distal fusiform aneurysm into the normal segment of the M1 (Fig 2B). The intervention was uncomplicated, and final angiographic runs demonstrated acute occlusion of the aneurysm neck without in-stent thrombosis and no further filling of the left A1 from a left ICA injection (persistent filling from a contralateral injection). The subject was maintained on dual antiplatelet therapy and had an uneventful course until postoperative day 5 when she developed a left MCA syndrome. Angiography revealed in-stent thrombosis (Fig $2 C$ ), and mechanical thrombectomy was performed using a direct aspiration technique with an aspiration catheter (ACE68) and a vacuum pump (ENGINE; Penumbra). In this intervention, the device was advanced over a microwire and microcatheter into an M2 branch using conventional endovascular techniques and then was directly connected to the vacuum pump. This maneuver resulted in a small amount of aspirated blood followed by absent blood flow into the canister. At that point, device pullback was performed under fluoroscopy, revealing severe collapse of the distal flow diverter, which resolved a few seconds after disconnecting the vacuum source (Fig 2D, $-E$ and Online Supplemental Data). This phenomenon was reproducible, even with the aspiration catheter located at the proximal cavernous segment of the ICA (Fig $2 F,-G$ and Online Supplemental Data). A final run after thrombectomy revealed complete recanalization.

\section{Arterial Collapse in the Rabbit Model}

All devices could be navigated into the aorta, and angiography demonstrated arterial patency without dissection or vasospasm (Fig $3 A,-B)$. A vacuum applied to the aspiration catheter resulted in stent-retriever collapse in $100 \%$ of cases (Fig 3C, $-D$ and Online Supplemental Data), followed by re-expansion after disconnecting the vacuum source. Collapse was confined to approximately $2 \mathrm{~cm}$ 

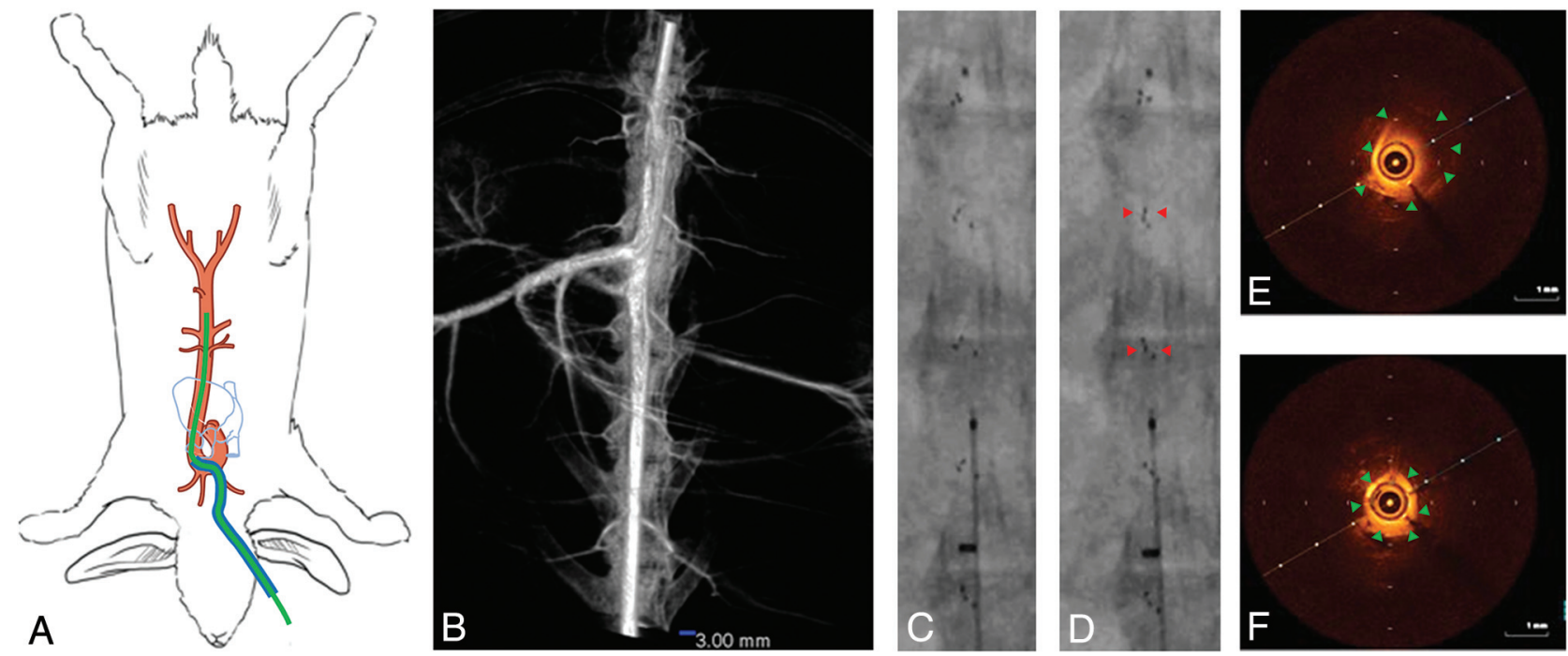

FIG 3. Aortoiliac rabbit model. $A$, Overview of the rabbit model with transcarotid aortic access. A 7F sheath (blue line) was advanced to the upper third of aorta, and a 5F aspiration catheter (green line) was coaxially advanced to the abdomen. B, 3D rotational angiography of a rabbit aorta. C, A stent retriever was delivered through a microcatheter and unsheathed to expand distal to a $5 \mathrm{~F}$ aspiration catheter. $D$, On activation of vacuum, the radiopaque markers of the stent came in close proximity, suggesting arterial collapse (arrowheads). The most distal fluoromarker did not collapse. $E$, Intravascular OCT imaging of the aorta depicted the arterial wall (with boundaries indicated by arrowheads) close to the probe, which becomes blurry on the opposite side of the circumference due to the scattering effect of blood. F, Vacuum applied to the catheter resulted in arterial collapse visualized by the whole circumference of the arterial wall (with boundaries indicated by arrowheads) in contact with the OCT probe.

of the arterial segment distal to the catheter front end. Arterial collapse was redemonstrated by removing the stent from the vasculature and introducing an OCT imaging probe distal to the aspiration catheter. The vacuum resulted in arterial collapse around the OCT probe (Fig 3E, $-F$ and Online Supplemental Data).

\section{Arterial Collapse in the Whole Human Brain Model}

The target pressure and flow within the cerebral vasculature were consistently achieved at baseline. The intra-arterial pressure in the M2 branches after temporary M1 ligation was $>60 \mathrm{~mm} \mathrm{Hg}$ at the high MAP condition, indicating patent leptomeningeal collateralization. Three data points were excluded due to a leak in the vacuum connection, and a total of 168 tests were available for analysis. We consistently observed a 20 - to $30-\mathrm{mm} \mathrm{Hg}$ pressure drop with the introduction of the suction catheter into the system. Connection of the catheter to the vacuum resulted in macroscopic deformation in $88 \%(147 / 168)$ and arterial collapse in $75 \%(126 / 168)$ of the cases.

In cases with macroscopic deformation, we identified 4 visibly discrepant stages: 1) indentation: focal inward movement of 1 side of the arterial wall closest to the catheter tip, sometimes resulting in low-amplitude reciprocal expansion and contraction of the lumen (Fig $4 A$ and Online Supplemental Data); 2) flutter: reciprocal cycles of focal collapse and re-expansion of the complete arterial lumen circumference distal to the catheter tip (Online Supplemental Data); 3) focal collapse: sustained collapse of a short segment of the whole arterial lumen into the catheter tip (Fig $4 B$ and Online Supplemental Data); and 4) full/extended collapse: complete collapse of a long segment of the artery extending beyond the catheter tip (Fig 4C, $-D$ and Online Supplemental Data). These 4 stages could be observed in the same vessel, and the deformation could transit from full collapse to indentation by gradually reducing the vacuum power. To compare the severity of arterial deformation with different catheters, locations, and flow conditions, we developed an arterial collapse score (ACS): ACS 1 for indentation, ACS 2 for flutter, ACS 3 for focal collapse, and ACS 4 for full collapse. Cases in which arteries remained unchanged had an ACS of 0.

The incidence rates of different arterial deformation stages and arterial collapse (focal or full) under aspiration thrombectomy for each combination of catheter-location-flow condition are presented in Fig 5 and the Table, respectively. Arterial collapse was consistently observed in $98 \%$ of the cases in the M2 $(n=52)$, regardless of catheter sizes and perfusion pressures. Arterial collapse was observed in all the cases with complete proximal flow arrest $(n=57)$. For the rest of the cases $(n=77)$, the probabilities of arterial collapse were $31 \%, 52 \%$, and $58 \%$ for the ACE64, ACE68, and Sofia PLUS catheters; 33\% and 59\% for the M1 and BA; and 34\% and 59\% for high and low MAPs, respectively.

The binomial logistic regression model included the catheter inner diameter ( 0.064 inch, $0.068 \mathrm{inch}$, and $0.070 \mathrm{inch})$, cerebral perfusion pressures (60 and $90 \mathrm{~mm} \mathrm{Hg}$ ), and location (M1 and BA) without data from cases with proximal flow arrest or tested in the M2. The Hosmer-Lemeshow test resulted in a $\chi^{2}$ value of 2.65 and a $P$ value of .96 and did not reject the null hypothesis of the model fitting the data. The binomial logistic regression model showed that a larger inner diameter of the catheter $(P=.034$, $\mathrm{OR}=1.246$; 95\% CI, 1.017-1.528), a lower $\mathrm{MAP}(P=.025, \mathrm{OR}=$ $0.31 ; 95 \% \mathrm{CI}, 0.116-0.867)$, and BA compared to M1 $(P=.022$, $\mathrm{OR}=0.309 ; 95 \% \mathrm{CI}, 0.112-0.847)$ were independent predictors of arterial collapse.

\section{DISCUSSION}

In this study, we report the second clinical case of arterial collapse during aspiration thrombectomy published to date, and 

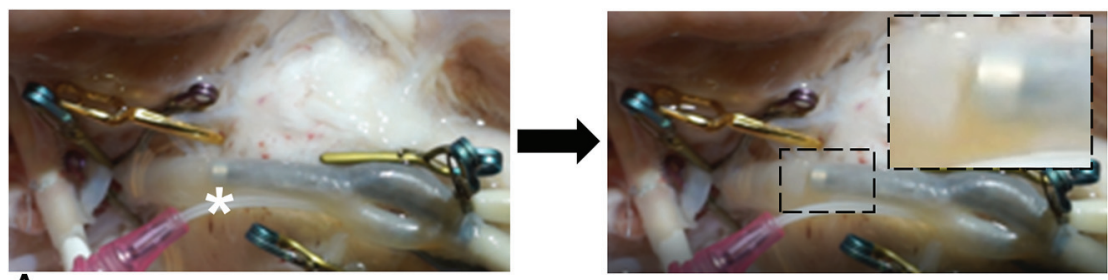

A
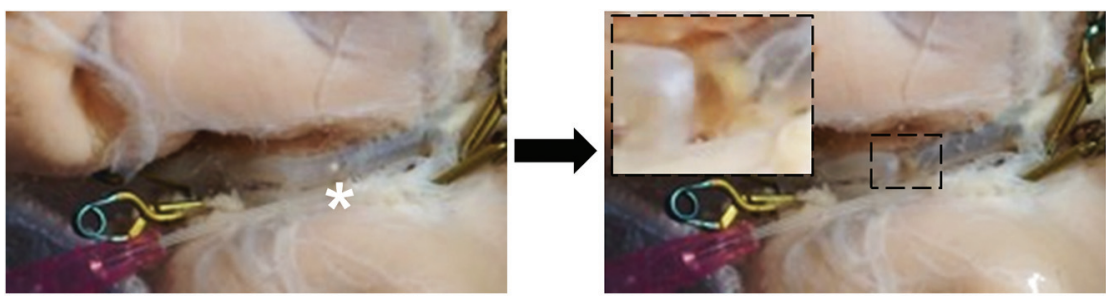

B
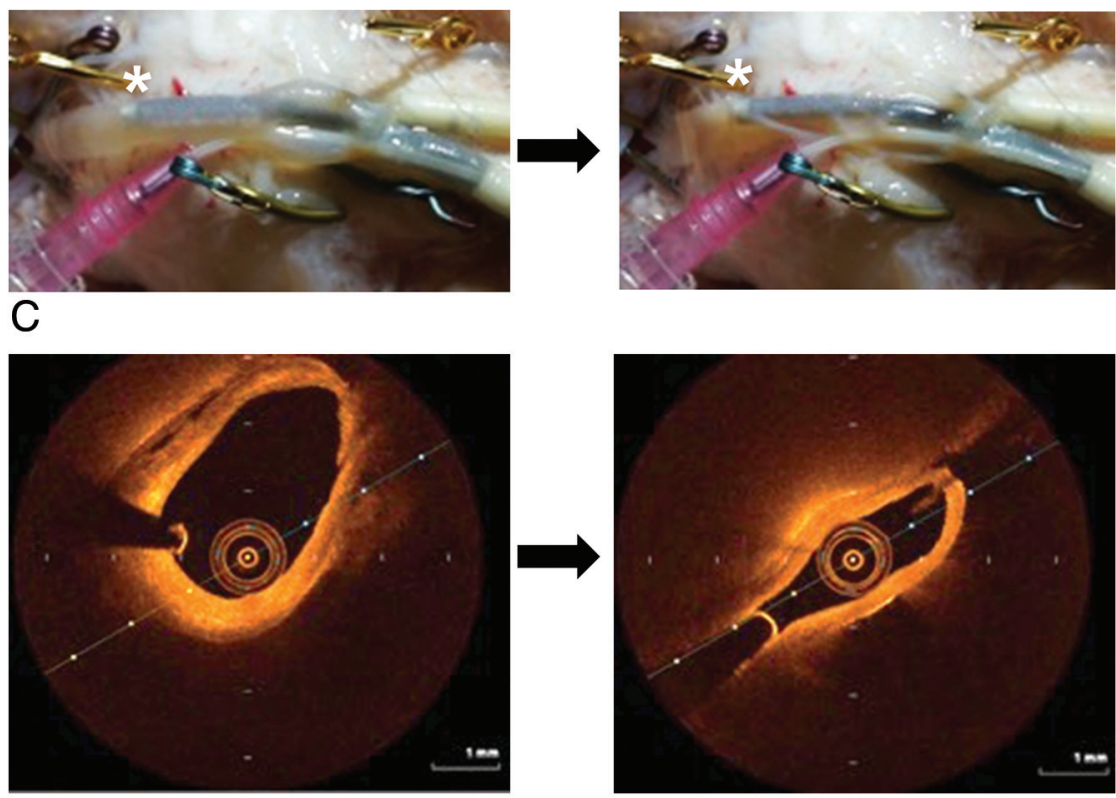

D

FIG 4. Different modes of arterial collapse in the brain model. $A$, Indentation the aspiration catheter tip (asterisk) in the BA. B, Focal collapse at the aspiration catheter tip (asterisk) in the MCA. C, Full/extended collapse of the BA spanning the catheter tip (asterisk). D, OCT shows a full collapse of the BA. we experimentally demonstrate the occurrence of arterial collapse in 2 different preclinical models. Dossani et $\mathrm{al}^{8}$ reported that aspiration using an 072 aspiration catheter in the right ICA causes collapse of a previously deployed radiopaque Neuroform Atlas Stent System (Stryker). Similar to this case, we were able to infer that arterial collapse had occurred, given the radio-opacity of the flow diverters previously implanted. Most interesting, collapse was observed in the most distal flow diverter in the MCA, even when the suction catheter was remotely positioned in the cavernous ICA. This finding suggests that the intraluminal negative pressure was transmitted throughout the "rigid" telescoping system (with 4 overlapping stents) for a long distance within the artery. Furthermore, it suggests that if the negative force was strong enough to collapse an artery buttressed with a high metal count stent (with 30\% metal coverage), it will likely be strong enough to collapse a compliant vessel without a stent.

To visualize the effect of vacuum in vivo under experimental conditions, we used a rabbit model previously developed and validated in our lab. The abdominal aorta of rabbits accurately represents the human large cerebral arteries with regard to the lumen diameter (approximately $3.5 \mathrm{~mm}$ ), mechanical properties, and flow speed. ${ }^{5}$ In our study, arterial collapse was inferred by the approximation of the stent retriever radiopaque markers and was directly visualized with intravascular OCT. Arterial collapse was consistently
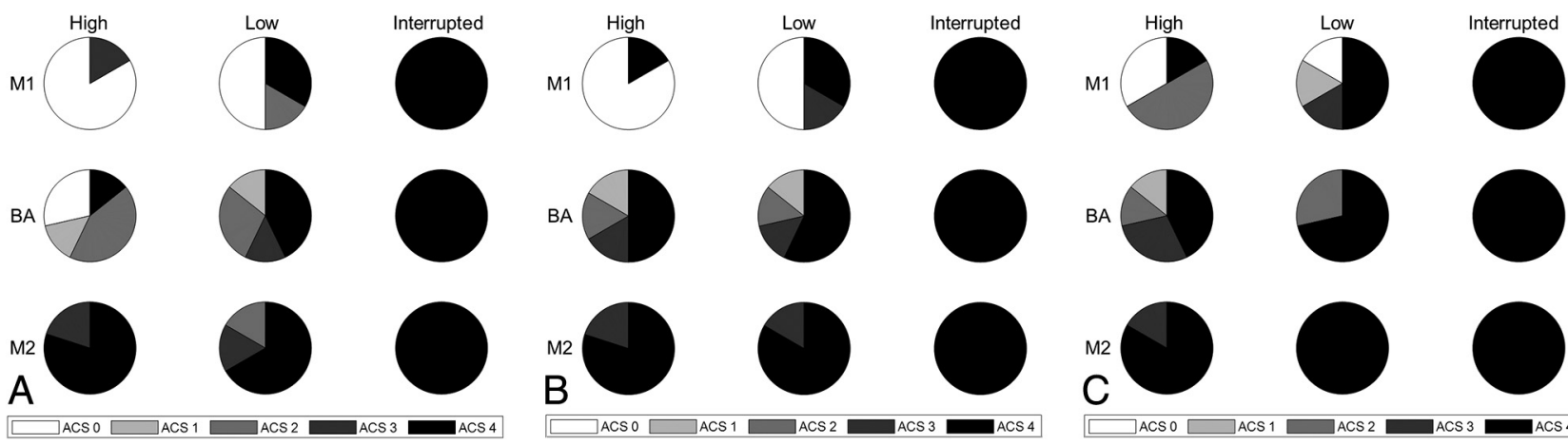

BA
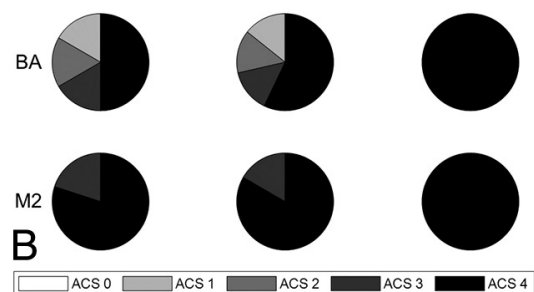
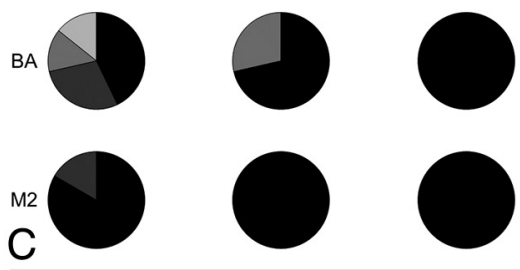

$\square$ ACS $0 \square$ ACS 1 $\square$ ACS 2 $\square$ ACS 3 $\square$ ACS 4

FIG 5. ACS result in the whole human brain model using 3 types of aspiration catheters: ACE64 (A), ACE68 (B), and Sofia PLUS (C). High indicates high mean arterial pressure; Low, low mean arterial pressure; Interrupted, proximal flow arrest. 
Incidence rate of focal or full arterial collapse in the human brain model using 3 aspiration catheters and under 3 flow conditions ${ }^{2}$

\begin{tabular}{lrrr}
\hline & High & Low & Interrupted \\
\hline ACE64 & & & \\
M1 & $17 \%$ & $33 \%$ & $100 \%$ \\
M2 & $100 \%$ & $83 \%$ & $100 \%$ \\
BA & $14 \%$ & $57 \%$ & $100 \%$ \\
ACE68 & & & \\
M1 & $17 \%$ & $50 \%$ & $100 \%$ \\
M2 & $100 \%$ & $100 \%$ & $100 \%$ \\
BA & $67 \%$ & $71 \%$ & $100 \%$ \\
Sofia PLUS & & & \\
M1 & $17 \%$ & $67 \%$ & $100 \%$ \\
M2 & $100 \%$ & $100 \%$ & $100 \%$ \\
BA & $71 \%$ & $71 \%$ & $100 \%$ \\
\hline
\end{tabular}

${ }^{a}$ High indicates high mean arterial pressure; Low, low mean arterial pressure; Interrupted, proximal flow arrest.

observed and generally confined to the arterial segment immediately distal to the suction catheter tip with luminal reconstitution more distally, likely from collateral flow.

To mechanistically validate the in vivo observations and to better understand the impact of different variables in the likelihood of arterial collapse by an experimental design, we used a validated human brain model. ${ }^{6,7}$ Unlike the other models using rigid or semirigid arterial phantoms, ${ }^{9-11}$ this validated model uses fresh human cerebral vessels (ie, flexible and collapsible arteries) and can reproduce different physiologic hemodynamic conditions. On connection of the catheter to the vacuum, we frequently observed collapse. This situation is likely due to a decreased anterograde flow, given the introduced catheter concurrent with brisk outflow of blood into the catheter due to the vacuum, reducing the intra-arterial blood pressure to negative levels between the catheter tip and the clot, resulting in arterial wall collapse. Although this situation could theoretically result in flow reversal and "noncontact" thrombectomy with migration of clots toward the suction catheter, we demonstrated that the pressure within the artery rapidly drops to negative values, resulting in arterial collapse. As expected, this phenomenon is more likely to occur at the tip of the suction catheter where the negative pressure is the highest.

Further supporting our hypothesis, we found that a larger catheter bore size is strongly correlated with higher collapsibility. Aspirational flow is proportional to the fourth power of the diameter of the catheter; therefore, small changes in catheter size (or target artery) would result in major increases in the aspiration flow along with a pronounced reduction in the blood inflow through the remaining arterial lumen. The larger the catheter is, the more violent the reduction in intraluminal pressure would be, resulting in more frequent and severe collapse.

On the basis of our findings, arterial collapse may occur within the range of hemodynamic conditions, even with the catheter sizes and vacuum levels generated by commercially available suction catheters; it could become a more serious event with the introduction of the newer generation of larger suction catheters such as the 088 catheter, which almost matches the size of the target artery.

We also found that lower perfusion pressure is correlated with higher collapsibility. This finding can also partially explain the higher collapsibility of the BA compared with the MCA. Although the BA is larger than the MCA, the inflow rate in the posterior circulation is lower than in the anterior circulation, thus potentially limiting the ability to overcome the aspiration flow generated by catheters. The results also showed that flow arrest using a balloon-guide catheter can cause full arterial collapse during aspiration thrombectomy.

Unfortunately, arterial collapse could be easily overlooked in clinical practice because the arteries are not visualized with fluoroscopy. The common teaching to recognize clot engagement in aspiration thrombectomy (with or without stent) is to turn on the vacuum and wait until there is no flow into the canister. On the basis of our findings, artery collapse would also result in a no-flow situation, which could be misinterpreted as integration with clots. As the artery collapses and hugs the catheter, the friction between the catheter and arterial wall can track the arteries. Such massive forces can denude the endothelial lining, create dissection flaps, and result in vasospasm. Moreover, arterial traction may result in avulsions, which could be an underlying reason for the higher rates of symptomatic intracranial hemorrhage reported in patients with more thrombectomy passes. ${ }^{12}$ Such forces can be further intensified when pulling an expanded stent retriever inside a collapsed artery. In older patients with cerebral atrophy, arterial collapse may occur more violently, given less perivascular tissue, thus leading to the adjacent branching vessels being damaged or even torn. Such a failure mechanism has been reported in ex vivo human brains. ${ }^{6}$ It has also been reported that the incidence rate of symptomatic intracranial hemorrhage was $>3$ times higher for M2 occlusions than for M1 occlusions. ${ }^{13}$ Our findings reveal that thrombectomy in the M2 branches results in more frequent and severe collapse and could, therefore, lead to microvascular avulsions during arterial traction.

Our experiments support the importance of advancing the suction catheter into or immediately proximal to the clot before connecting to the vacuum source to mitigate the risk of arterial collapse and possible no-flow situations, and they suggest that for the case of suction catheters, larger is not always better. The selection of the catheter size should be a thoughtful process considering multiple variables including parent vessel size, the configuration of the circle of Willis and collaterals, and the MAPs. Future suction-based technology should be developed to enable more efficient clot removal at equal or lower vacuums and smaller catheter size and/or to inform the interventionalist about the clot/device/artery interaction.

There are a few limitations we would like to acknowledge. First, in this study, clot analogs were not used for either the animal or the ex vivo human brain tests. Because clots are generally corked at the catheter tip reducing (or completely stopping) the amount of blood outflow, the intraluminal pressure is unlikely to drop to negative levels resulting in collapse. The testing here conducted without clots can mimic the cases in which aspiration continues after the clot is ingested or when the catheter is not in direct contact with the face of the clot. The failure modes of mechanical thrombectomy including arterial collapse in a human brain model with intraluminal clots have been recently published. ${ }^{6}$ Second, the collateral circulation of the rabbit's aorta is not well-characterized, and extrapolation to human cerebral arteries needs to be done with care because arterial collapse might be overestimated. Third, although pressure and flow are closely measured and controlled during the experiments in ex vivo human brains, the reduced vascular tone and mechanical constraints from perivascular tissue could make the arterial wall 
more prone to collapse under a vacuum. Additionally, in the human brain model, flow interruption is truly complete in comparison with the clinical use of balloon-guide catheters. These are usually placed in the cervical or petrous ICA and, therefore, do not compromise the inflow from the ophthalmic artery and other collateral pathways. Fourth, the ACS scale was developed on the basis of testing in ex vivo human brains and may not translate to patients. Validation in patients using different sensing technologies or techniques that can monitor the mechanical response of arteries in real time is needed. Finally, the use of saline solution instead of blood (which has a higher viscosity) could result in higher aspiration flows leading to more easily reduced intraluminal pressure. Therefore, translation to clinical care needs to be interpreted with caution.

\section{CONCLUSIONS}

Aspiration thrombectomy can result in arterial collapse both clinically and in preclinical experimental models and is directly associated with larger-bore catheters, lower blood pressure, and a smaller target artery.

\section{ACKNOWLEDGMENTS}

We thank D. French, C. Prescott, D. Griffiths, and J. Jentzen for their expertise and technical assistance in obtaining human cadaveric specimens. We would also like to thank the Department of Laboratory Medicine and Pathology and the Tissue Request Acquisition Group at the Mayo Clinic, Rochester, for their valuable assistance in obtaining human brains.

Disclosure forms provided by the authors are available with the full text and PDF of this article at www.ajnr.org.

\section{REFERENCES}

1. Guo X, Miao Z. Advances in mechanical thrombectomy for acute ischaemic stroke from large vessel occlusions. Stroke Vasc Neurol 2021 Jul 20 [Epub ahead of print] CrossRef Medline

2. Lapergue B, Blanc R, Gory B, et al; ASTER Trial Investigators, Effect of endovascular contact aspiration vs stent retriever on revascularization in patients with acute ischemic stroke and large vessel occlusion: the ASTER randomized clinical trial. JAMA 2017;318:443-52 CrossRef Medline

3. Turk AS, Siddiqui A, Fifi JT, et al. Aspiration thrombectomy versus stent retriever thrombectomy as first-line approach for large vessel occlusion (COMPASS): a multicentre, randomised, open label, blinded outcome, non-inferiority trial. Lancet 2019;393:998-1008 CrossRef Medline

4. Almandoz JE, Kayan Y, Wallace AN, et al. Larger ACE 68 aspiration catheter increases first-pass efficacy of ADAPT technique. $J$ Neurointerv Surg 2019;11:137-40 CrossRef Medline

5. Ding Y, Fitzgerald S, Liu Y, et al. A novel rabbit thromboembolic occlusion model. J Neurointerv Surg 2021;13:1040-43CrossRef Medline

6. Liu Y, Gebrezgiabhier D, Reddy AS, et al. Failure modes and effects analysis of mechanical thrombectomy for stroke discovered in human brains. J Neurosurg 2021 Jun 4 [Epub ahead of print] CrossRef Medline

7. Gebrezgiabhier D, Liu Y, Reddy AS, et al. A human brain test bed for research in large vessel occlusion stroke. J Neurosurg 2021 Jan 22 [Epub ahead of print] CrossRef Medline

8. Dossani RH, Tso MK, Waqas M, et al. Mechanical thrombectomy of right internal carotid artery terminus occlusion using the ADAPT technique: impact of aspiration on parent vessel. J Neurointerv Surg 2020;12:1148 CrossRef Medline

9. Liu Y, Zheng Y, Reddy AS, et al. Analysis of human emboli and thrombectomy forces in large vessel occlusion stroke. J Neurosurg 2020;134:893-901 CrossRef Medline

10. Reddy AS, Liu Y, Cockrum J, et al. Construction of a comprehensive endovascular test bed for research and device development in mechanical thrombectomy in stroke. J Neurosurg 2020;134:1190-97 CrossRef Medline

11. Liu Y, Abbasi M, Arturo Larco JL, et al. Preclinical testing platforms for mechanical thrombectomy in stroke: a review on phantoms, in vivo animal, and cadaveric models. J Neurointerv Surg 2021;13:81622 CrossRef Medline

12. Maros ME, Brekenfeld C, Broocks G, et al. Number of retrieval attempts rather than procedure time is associated with risk of symptomatic intracranial hemorrhage thrombolysis in cerebral infarction. Stroke 2021;52:1580-89 CrossRef Medline

13. Saber H, Narayanan S, Palla M, et al. Mechanical thrombectomy for acute ischemic stroke with occlusion of the M2 segment of the middle cerebral artery: a meta-analysis. J Neurointerv Surg 2018;10:62024 CrossRef Medline 